\title{
Telemedicine and the Academic Health Center: The University of Michigan Health System Model
}

\author{
GARY W. SHANNON, Ph.D., ${ }^{1}$ RASHID BASHSHUR, Ph.D., ${ }^{2}$ \\ ERIC KRATOCHWILL, M.H.S.A., ${ }^{2}$ and JOCELYN DEWITT, Ph.D. ${ }^{2}$ \\ " . . reliance on our own productivity to fund needs and aspirations." \\ _UMHS Strategic Direction, 2005-2010
}

\begin{abstract}
The changing health care environment is generating a number of challenges for Academic Health Centers' (AHC) ability to pursue their traditional tripartite mission of medical care, education, and research. A number of strategies have been suggested to aid the AHC to respond, among them telemedicine. In this paper, telemedicine is examined for its potential to assist in meeting financial, cost, and quality challenges. In particular, the model developed at the University of Michigan Health System for implementing telemedicine within the AHC is presented together with lessons learned. This model, based largely on intramural rather than extramural funding, is offered as a basic strategy to be considered by AHCs facing these challenges.
\end{abstract}

\section{INTRODUCTION}

$\mathbf{T}$ HE PURPOSE OF THIS PAPER is three-fold. The first is to describe the challenges pertaining to utilization, cost, and quality facing academic health centers (AHCs) and the potential role of telemedicine in meeting these challenges. Unless otherwise specified "Telemedicine" refers to and includes telehealth, e-health, and medical informatics. In this broader context, the specific challenges facing the University of Michigan Health System (UMHS) will be illustrated. Second, within the framework of this perspective, the initiation, development, and deployment of telemedicine at the UMHS will be described. Finally, suggestions are offered for the future development of telemedi- cine to assist AHCs generally and the UMHS specifically in helping to meet the typical AHC mission.

It is hoped that the description of the UMHS experience with telemedicine development, accomplished largely through institutional support for meritorious faculty initiatives and intramural funding, may serve as a model for other AHCs considering initiating or expanding their own telemedicine programs to meet the challenges of a changing health-care environment.

Increasing geographical and temporal access to medical care, assuring and enhancing the quality of medical care and containing rising costs of medical care, coupled with rapid strides in information technology have served

\footnotetext{
${ }^{1}$ Department of Geography, University of Kentucky, Lexington, Kentucky.

${ }^{2}$ University of Michigan Health System, Ann Arbor, Michigan.
} 
as the rationale and basis, respectively, for the development and implementation of the vast majority of telemedicine programs in the United States and elsewhere. However, when examined closely, no two programs are exactly the same in terms of their clinical and nonclinical applications, equipment in use, and financial support. In terms of organizational affiliation, however, according to information available from the Telemedicine Information Exchange, ${ }^{1}$ most large-scale telemedicine programs in the United States are affiliated with AHCs. Furthermore, the perspective of the AHC with regard to telemedicine may contrast considerably, for example, with those of a district or community hospital, federal (civilian or military) agency, private company, or nonprofit research institute.

\section{ACADEMIC HEALTH CENTERS}

There are some 125 AHCs in the United States accredited by the Association of American Medical Colleges, most based on partnerships-between universities and hospitals, and between university-hospital systems and state as well as local governments. Although diverse with regard to their organization, structure, size, and operation, the AHCs are similar in regard to their missions. ${ }^{2}$ More specifically, the AHCs serve three essential roles simultaneously: (1) to provide medical education and training, (2) to conduct biomedical and clinical research, and (3) to provide direct patient care. Typically, their missions emphasize the training of the best physicians, conducting state-of-the-art medical research, and providing optimal patient care for serious and complex medical conditions. Moreover, it is widely recognized and acknowledged that an appropriate relationship and balance between the three roles is necessary.

There is a substantial recent literature pertaining to various threats to the AHC missions as a result of the changing health-care environment. ${ }^{2-4}$ These threats revolve around financial problems resulting from expenses outpacing revenues, a trend of increasing regulations and restrictions on reimbursement, as well as rising consumer expectations. Unfortunately, much like the situation regarding telemedicine evaluation research, as Freburger and Hurley ${ }^{2}$ point out, our understanding of the AHC is generally limited due to the "anecdotal, speculative, or descriptive nature" of the majority of the literature in the field. Furthermore, research on threats to AHCs as well as strategies adopted by AHCs in response to a changing health care market suffer from lack of sound empirical research due to the complexity of the issues and the limited potential for in-depth research to reveal the dynamic and changing nature of the health-care environment. As well, the heterogeneity among AHCs in terms of structure, finance, and operations, the lack of timely, relevant, and sensitive performance measures for each of their components, as well as location in markets with varying degrees of competition, contribute to difficulties in assessing and generalizing the threats to and responses/adaptations of the AHCs in the face of these challenges to their missions.

For example, an essential AHC mission, patient care, is complex in its own right. Several critical issues facing the AHC pertain to patient care, notably, service and patient mix, level of specialization, and volume. The patient mix and volume must be appropriate, not only for this specific charge of the AHC but also for medical education and research as well. Therefore, the question of which patient mix to be seen at the AHC and the accompanying question of patient volume can affect the quality of and advances in care as well as medical education and research.

Of course, one of the major responsibilities of the AHC is to treat serious and complex medical conditions. Due to the complexity of these cases, the inherent difficulties in treating them, and the sheer size and complexity of these centers, quality is occasionally compromised. The Institute of Medicine (IOM) has identified four major types of errors contributing to substandard quality in these instances, and has offered recommendations for defining serious or complex medical conditions for consideration by health plans. ${ }^{5}$ Although identification of these errors and recommendations were directed toward health-care plan devel- 
opment, they are instructive in evaluating both the tripartite mission of the AHC and the potential role of telemedicine in achieving the mission goals.

The types of errors identified by the IOM included avoidable errors and underutilization and overuse of scarce services. As well, errors associated with regional and small-area variation in health-care practices and protocols were suggested as contributing occasionally to less than optimal care. The AHC is, of course, no exception to the general rule that avoidance/minimization of error is essential to optimal care.

With regard to the errors listed here, telemedicine, broadly defined, can assist the $\mathrm{AHC}$ in the following ways:

- It can decrease the incidence of avoidable errors by improving medical record keeping and access to such records; improving the prescribing and dispensing of drugs; and, monitoring drug therapy as well as providing computer-assisted practice guidelines and information on demand. Though still in early stages of development, robotic surgery can decrease human error through greater precision.

- It can decrease underutilization of specialized and general medical care resources through improved timely access (by decreasing the need for travel for remote and isolated and home-based populations) as well as diminishing appointment delay time and office waiting time.

- It can diminish inappropriate use or overuse of scarce specialty medical services through triaging patients to appropriate medical care facilities for initial diagnosis and treatment and post-hospitalization and post-operative follow-up care.

- Finally, it may also decrease regional and small-area variation in medical practice as a result of greater interaction between primary and secondary practitioners on the one hand and specialist consultants in academic medical centers on the other; and through immediate access to current literature on practice guidelines, protocols and standards of care in various clinical areas; and just in-time continuing medical education.
The health and general welfare of society in the long-term is best served by devoting scarce and specialized AHC resources to serious and/or complex medical conditions. To this end, the IOM also recommended identification of individuals with serious or complex medical conditions; assessment of these conditions, including medical procedures to diagnose and monitor them on an ongoing basis; and the establishment and implementation of a treatment plan appropriate for these conditions, with an adequate and appropriate (authors' emphasis) number of direct access visits to specialists to accommodate the treatment plan.

The inpatient and outpatient admission policies must be designed to reflect the priority of these concerns. The AHC must safeguard against underutilization by patients with serious and complex conditions requiring specialty expertise and advanced facilities available only at the AHC and, simultaneously, overutilization by patients with more common conditions. Optimally, AHCs would find the appropriate balance through their triage and admission policies that promote the admission of patients with serious and complex medical conditions requiring the range of expertise, services, and equipment unique to the AHC. The volume and variety of conditions among the patients must be sufficient to meet and reflect the requirements for the medical care, education, and research missions of the AHC. At the same time, the less serious and routine medical conditions necessary for physician training and education can be derived from selective inpatient and outpatient admission to the major AHC facilities and its distributed network of medical clinics.

It has been suggested that the general population's access to highly specialized care is inconsistent, and this may result in a corresponding lack of access by physicians to enough complex and difficult cases for them to develop their expertise. ${ }^{6}$ For example, patients with unstable asthma might end up being treated in their community hospital when optimally they should be seen by a pulmonologist in a specialized center. At the same time, without control of patient mix and flow, the pulmonologist is quite likely seeing large numbers of moderate asthmatics who do not require the service of a specialist. The individual 
with unstable asthma might be referred as an outpatient to a teaching hospital, and thereby gain access to specialist care. But this process does not allow for concentrated access to these patients by physicians, which is needed for specialists to acquire competence.

Primary among a number of identified threats to the provision of patient care, education, and research at AHCs is for-profit managed care. ${ }^{2,4,7}$ The perceived threat by many AHC administrators is reflected in Eisenberg's rather dire suggestion of a corollary to Gresham's Law that states "bad money drives out good," namely, that "for-profit managed care drives out care, teaching and research." ${ }^{7}$ A concern critical to the AHC and the completion of its missions is, of course, maintaining fiscal integrity and solvency. This too relies to a certain extent on the volume and mix of patients required by the AHC's teaching and research missions. It has been suggested that when this condition is not met, the AHC must work with competing health plans in the relevant market area to attract the requisite volume of patients. ${ }^{8}$

An additional fiscal concern rests on the fact that, traditionally, the nation's AHCs have been a major source of health care for the uninsured. For millions of low-income and uninsured Americans, the AHC has become a major source of charity care. The question facing many AHCs is how they can continue to finance their traditionally large and often increasing volume of charity care while meeting their specialized care, education, and research charges. ${ }^{2}$ This situation may, in some instances, drive the AHC away from specialty training to primary care training-even if only to sustain revenues. ${ }^{8,9}$ If this were to occur, it would have major negative short- and long-term consequences for patients and the community, as well as for the progress of scientific medicine. On the other hand, some suggest that the future of the AHC may rest in further demarcating themselves as centers of excellence and quaternary care. ${ }^{10} \mathrm{But}$, it is also recognized that the AHC cannot survive on providing quaternary care alone. ${ }^{3}$

Concern for the challenges facing the AHC continues, and several approaches have been identified recently for exploration by AHCs to compete effectively. ${ }^{4}$ These pertain to cost, comprehensiveness, capacity, coverage, continuity, communication, coordination, consistency, and consolidation. Also, several strategies have been recommended for AHCs to "push back" against the challenges derived from managed for-profit care, which include decreased patient care revenue, increased numbers of uninsured, increased competition on the basis of price, and increased cost of technology. ${ }^{3}$ Some strategies address the problems with managed care, most of which are already in place in different degrees at many institutions. Other strategies include demonstrating that the AHC is "different" or "better" and provides a better quality of care through improved effectiveness and efficiency of patient care; having centers for health services research; implementation of quality improvement programs; development of centers for clinical research to create protocols for efficient patient care and outcome analysis; improved scheduling in all departments and centers; the use of Web-based technology to monitor and care for patients at home; controlling market share in one or more service areas through cooperation of quaternary and tertiary hospitals; and, expanding the size and location of their physician referral networks.

Regardless of the proposed solution or strategy, it is imperative that the AHCs operate more efficiently and cost effectively. In this context, it was observed in 1999 that among "other strategies that have been suggested, but have not been well adopted yet, are the use of telemedicine, the development of integrated computer systems, and the use of physician extenders." 2 Since that date, a good number of AHCs have adopted telemedicine as one of the measures to meet the many challenges facing them. However, the approaches in funding, designing, and implementing these systems vary considerably from place to place. Additionally, the specific role and relative effectiveness of telemedicine in meeting challenges may be specific to each AHC.

Interestingly, AHCs can be grouped into one of several larger categories in terms of the relative importance of specific challenges facing them. For example, one group might be faced with low bed census rates and declining outpatient visits while another may have bed cen- 
suses approaching capacity and excess demand for outpatient services. Similarly, AHCs vary with regard to uncompensated visits and an inappropriate mix and level of cases. Hence, to succeed, each AHC must evaluate its particular challenges and provide an appropriate response, including the type of telemedicine most suitable for its circumstances and needs. One solution may not fit all. At the same time, however, there may be general lessons to be learned and directions to be considered by AHCs from the experience of other AHCs with telemedicine to date. To this end, in the remainder of this paper, we describe briefly the organizational structure and mission of the UMHS, its hospitals and health clinics, and the role and contributions of telemedicine within that structure. The central objective is to draw some lessons and conclusions with regard to a sustainable and viable telemedicine system within an academic health center.

\section{THE UMHS}

The UMHS consist of four entities: (1) the Medical School and its Faculty Group Practice (FGP), (2) the Hospitals and Health Centers (HHC), (3) the M-Care Health Plans, and (4) the Michigan Health Corporation (MHC).

The Medical School first opened its doors for students in 1850. It was the first medical school in the United States to own and operate its own hospital, and among the first major medical schools to admit women and to base its instruction on science. The Medical School was also a pioneer in the introduction of the modern medical curriculum and the development of the clinical clerkships as part of medical education. Today it has a student body of 680 undergraduate and 958 residents and fellows. Research expenditures by its faculty totaled $\$ 289$ million in 2003.

The HHC includes three hospitals-University Hospital, Mott Children's Hospital and Women's Hospital. Together, they have 865 licensed beds spread over 2,135,816 square feet, and admit more than 41,000 patients annually. The HHC also includes the Taubman Outpatient Health Care Center and its 120 outpatient clinics; Emergency Services; the Comprehensive Cancer Center and Geriatrics Center; numerous specialty, rehabilitation, substance abuse treatment, cancer detection, geriatrics and sports medicine facilities; a system of 30 community-based health centers; and the Kellogg Eye Center. The HHC serve more than 1.5 million outpatient visits each year.

The M-CARE health plan has grown from its roots at the University of Michigan to become one of the State's leading health care plans. It offers a variety of health insurance coverage options for its members including HMO, POS, PPO, and HSA plans. It serves more than 200,000 members across Southeastern Michigan and nearly 1,500 employer groups.

The MHC is a separate corporation established by the University for joint ventures and special projects that support the UMHS mission. It provides UMHS with the ability to enter into partnerships with other health-care organizations and businesses. MHC has full or partial ownership interest in nine companies that provide direct health care or health care business support services throughout the state.

The UMHS continues to anticipate the resources needed to pursue its tripartite mission of education, research, and patient care. Its "Strategic Direction" plan outlines broadbased growth in research and clinical services, with larger growth in priority areas defined by strategy. Such growth is facilitated by investment in human and physical resources. Major construction projects include the Children's and Women's Hospital Replacement; the Cardiovascular Center; the Depression Center; the East Ann Arbor Ambulatory Surgery and Medical Procedures Center; the Biomedical Science Research Building; and a new Ophthalmology and Diabetes clinical and research center.

\section{UMHS MISSION AND STRATEGIC GOALS}

As with other AHCs, the UMHS mission has three critical components-patient care, education and research, which, in synergistic combination, enhance (its) contributions to society. ${ }^{11}$ To this end, the UMHS has set goals and strategic targets in each sector for 2005-2010. Among the goals and targets that pertain directly to implementation of information technologies, including telemedicine, are the following. 


\section{Educational goals}

The UMHS has explicit goals with regard to the use of information technology to advance education and training:

- Continuing to develop and employ innovative clinical technology in simulation learning. The Simulation Center provides a critical resource for clinical training and education.

- Using information technology and e-health to create opportunities for patients and their families with immediate access to appointment scheduling and billing as well as just-intime educational materials, including standardized treatment plans and protocols. Similarly, providers would have immediate access to patient records, as needed, for their care. When appropriate and necessary, the records would also be readily available to authorized family members, referring providers, students, residents and other faculty.

\section{Patient care goals}

Similar to education, the UMHS has established fairly explicit goals in terms of using information technology to advance service to patients and their families. The following general goals are not specific to telemedicine, but point to the general orientation of the institution with regard to the use of information technology. The goals include the following

- To improve the health of the community and the service population by meeting the growing demand for service in a cost-effective manner

- To develop a partnership with patients, their families, referring providers, and community, strengthened by clear and honest twoway communications

- To increase patient care activity (inpatient and outpatient) by at least 3\% per year, with faster growth for high priority clinical areas (authors' emphasis)

- To improve access to care for patients from faculty, referring providers, and emergency services

- To employ new technologies and practice changes to continue the trend for inpatient to outpatient to home-care services
- To implement an integrated, fully accessible electronic patient care record before 2010, beginning with implementation of the Orders Management Project for all UMHS patients by 2006 .

The goals that pertain to telemedicine and ehealth specifically include the following:

- Further development of the electronic order entry, result reporting, and electronic medical records

- Using telemedicine to supplement consultations and care in patients' homes and in areas lacking sufficient clinical specialties

- Establishing computer links with patients, referring providers and other health-care professionals

- Providing for remote and portable diagnosis and treatment, including biosensors, measurement devices, and administration devices

- Enabling Web-based shopping for medical care, medical equipment, supplies, medication, information, and other services, with resulting financial and quality implications

- Providing expert systems to analyze, simulate, and support decisions.

It is clear that these goals are not mutually exclusive. For example, increasing patient care activity is coupled with a desire for faster growth in "high-priority clinical areas." In turn, this relates directly to the teaching and research missions. And, the use of telemedicine to supplement consultations and care by providers in patients' local communities may serve to prevent unnecessary initial visits as well as follow-up visits to the UMHS facilities. This strategy allows UMHS clinicians to utilize the available inpatient and outpatient capacity more efficiently.

\section{OPERATIONAL CHALLENGES FACING THE UMHHC}

In this section, the general status of the University of Michigan Hospitals and Health Centers (UMHHC) vis-à-vis the suggested challenges facing AHCs is discussed. 


\section{Patient volume}

UMHHC inpatient activity as measured by bed occupancy reflects a situation of stress on the capacity of the system. Through April of 2005 , the average daily occupancy rate was $89.1 \%$ with a projected annual forecast of $93.4 \%{ }^{12}$ Periodically, and on a temporary basis, daily occupancy rates rise to over $100 \%$. Acute-care hospitals must balance immediate patient care needs while having the capacity to meet urgent and emergent needs for hospital beds. To allow this flexibility, research has suggested that the "ideal" acute-care hospital occupancy rate is approximately $85 \% .{ }^{13}$ Although historical increases in average patient acuity, advances in technology, and other factors continue to contribute to the debate of ideal occupancy rates, it is generally accepted that excessive occupancy rates contribute to stress on the personnel, systems, and facilities that support patient care and may impact quality. ${ }^{14}$ Occupancy rates above $90 \%$ place unusual stress on the staffing of direct patient care givers, the timely provision of diagnostic and therapeutic services, as well as the maintenance of nonclinical support systems for patient care. Furthermore, high occupancy rates may result in regular bed crises that contribute to faculty, staff, and patient stress and dissatisfaction.

Since 1997, the annual growth rate of inpatient discharges has ranged from $2 \%$ to over $4 \%$. Over the past 5 years, the average annual growth in inpatient discharges has been 3.1\% and $2.7 \%$ in outpatient visits. The 10 -year fiscal and activity model projects an average annual growth of $3 \%$ increase in inpatient volume and $2.3 \%$ in outpatient visits. On the other hand, the experience with length of stay has been mixed. For example, from 2000 to 2003, length of stay declined from 5.77 to 5.48 days, but it increased to 5.64 days in 2004, and increased to 5.74 days in 2005. Ninety-nine percent of operating budget for the UMHHC is derived from patient care.

During the period extending from fiscal year (FY) 2002 through the first quarter of FY 2005, the percentage of transfer cases from other facilities has declined. This may not be due to the changing health-care environment but to the increase in occupancy rates, which reduced the
UMHHC's ability to accept some transfers. However, this trend has changed, and the complexity and severity of illness of transfer cases has increased since FY 2002. Furthermore, as might be expected, the complexity and severity of illness of the transfer cases, as measured by the APR-DRG case-mix index, far outweighs that of nontransfer cases. This means that considerably more resources are needed to treat transfer cases, some of which are made more difficult due to delay in treatment.

Defined narrowly as bad debts and charity cares, the UMHHC uncompensated care rates remained relatively consistent (at $2 \%$ ) from 2000 through 2002. During the past 3 years, the percentage decreased to an estimated figure reaching $1.4 \%$ for first quarter of 2005 . What these data do not reflect, however, are increases in patients covered by state and county health insurance plans, which reimburse hospitals at rates below cost. Although some studies suggest that public AHC hospitals in particular have negative operating margins or that operating margins have been decreasing, the UMHHC has increased its operating margin substantially over the past 5 years. The UMHHC operating margin increased from $\$ 5.5$ million in 2000 to over $\$ 70.7$ million by the first quarter in 2005. As a percentage of total operating revenue, it increased from 1\% in 2000 to $5.4 \%$ during the same period. The UMHS relies upon this growth to pursue its plans for broadbased support for research and clinical services, with larger growth in priority areas defined by strategy.

\section{UMHS TELEMEDICINE}

It is in the context of current and projected growth of the institution and the increasing demand for service, the increasing severity and complexity of transfer cases, and increasing percentages of uncompensated and under compensated care that telemedicine is being considered to meet these challenges. Unlike other academic health centers that relied on extramural funding for telemedicine initiatives, the UMHS chose to fund telemedicine internally so that the investment would specifically address these institutional challenges. 
One of the most visible signs in support of telemedicine in the strategic goal pertaining to telemedicine specifically was the establishment of The Telemedicine Resource Center (TRC) in 1998. This was accomplished largely at the initiative of the Chief of Clinical Affairs, with strong support from the Chief Executive Officer of the UMHHC, the Dean of the Medical School, and the Dean of the School of Public Health. Accordingly, a Director of Telemedicine was appointed, together with support staff, for the purpose of organizing and supporting telemedicine initiatives at the UMHS; assisting in the development of strategic and business plans for telemedicine initiatives; supporting faculty and staff in pursuing their interests in telemedicine research and development; and, in assisting faculty and staff to procure extramural research and development funding when opportunities present themselves. However, the bulk of the TRC activities relate to internal initiatives and programs.

Early in the development of the TRC, the UMHS provided substantial funding for faculty initiatives in telemedicine with active support and oversight by the TRC. These funds were provided as part of a larger program of intramural investment in innovative and potentially beneficial initiatives proposed by faculty and staff. As part of its function, the Faculty Group Practice (FGP) at the UMHS has managed this investment fund and set aside funds to support initiatives that promise to improve quality or efficiency of patient care and provide a potential positive return on investment. Competition for these awards has been open to all faculty and staff, and the process for allocating funds is similar to those established by foundations or federal agencies. Hence, formal proposals are required that identify the objectives of the proposed project, description of the methods of implementation, expected outcomes, budget, and staffing. The total amounts of funding available as well as the funding cycles are determined by the availability of special-purpose funds.

During the TRC's initial year of operation, several proposals were submitted pertaining to various telemedicine applications, four of which were funded. These are described below. The TRC was asked to assist in the develop- ment, review, and prioritizing of the proposals and, once funded, to coordinate, monitor, and provide technical support for these projects. Funds were provided through the TRC, and project directors were required to provide progress reports to the TRC. A standing committee consisting of the principal investigators of these projects and the director of telemedicine was established. The committee held regular meetings to review the progress in the implementation of these projects, problems encountered, and responses to the problems that emerged.

The first project was a pediatric emergency service to provide specialist teleconsultations for several remote sites. The most notable site was Marquette General Hospital (MGH) located in the central Upper Peninsula of Michigan on the shores of Lake Superior. In turn, the MGH operates as a central hub of its own telemedicine network in the Upper Peninsula. A formal agreement was concluded between MGH and the UMHS that defined the terms and conditions of collaboration between the two institutions. In addition to $\mathrm{MGH}$, this project provided on-going pediatric echocardiography service for interested providers throughout the State of Michigan.

The second project was dedicated to child and adolescent psychiatry. In this project, the UMHS entered into a formal agreement with the Chippewa Mental Health Center, a mental health clinic in the located in Sault Ste. Marie in the eastern Upper Peninsula of Michigan, to provide psychiatric teleconsultation services for the Center's child and adolescent patients during regularly scheduled clinic hours on certain days of the week. ${ }^{15}$ At the conclusion of the initial funded stage of this project, the Chippewa Health Clinic requested the continuation of the program on a contractual basis at its expense, and the project was accordingly extended.

The third project employed digital photography and formal protocols in the management of chronic wounds and pressure ulcers in the home environment. This project was conducted jointly by the UMHHC Division of Plastic Surgery (DPS) and the Michigan Visiting Nurse program, which is formally affiliated with the UMHS. The project also pro- 
vided education and training, with the TRC providing coordination and ongoing technical support. This project is currently being expanded to include a comprehensive teleconsultation program for wound management that incorporates both inpatient and outpatient services. Arrangements are underway to extend the scope and to secure the continuity of this project.

The fourth project funded during the initial year consisted of home-based monitoring of hypertensive patients, which focused on the development of technological tools for such a service, rather than the provision of services. This was done jointly with the School of Engineering.

Support for development and implementation of other projects was not limited to funding from the FGP, because additional funding was made available on the basis of special requests by the faculty at the UMHS. Some of these projects received financial and logistical support from UMHHC operational and capital funds, some on an on-going basis. The projects included the following:

1. A remote system to support telepsychiatric court hearings with the District Court in Washtenaw County for commitment of mental patients. This project uses a secure ISDN line between the District Court and the inpatient psychiatric unit in the hospital. It obviated the need for transporting mental patients together with clinical and support staff from the hospital facility to the court, thereby eliminating the cost, long wait, and the security risks associated with these trips. Plans are underway to extend this service to other courts within the area.

2. A special research project was initiated jointly with the Department of Dermatology aimed at improving timely access to care and reducing long waits for appointments through a store-and-forward triage telemedicine system. More specifically, it was aimed at expediting appointments for critical cases and deferring others to their usual providers. Financial support in the form of equipment purchase, training manuals, and protocols were provided for a teledermatology triage pilot project. The intervention was based on digital pho- tography and the use of a simplified referral protocol.

3. A large-scale home-based monitoring system for congestive heart failure patients by the Department of Cardiology is being developed in cooperation with the Heart Failure Disease Management Program and the Medical Management Center. The project recently submitted a formal application for extramural funding to support collaboration with patients in the design of a new chronic illness monitoring system. Following its successful completion, it is hoped that the model will be extended to other chronic diseases.

4. In response to a request from the Department of Dermatology, the TRC designed and supported a project for Mohs surgery referral, integration, and follow up. The Mohs surgeons remove basal cell and squamous cell carcinoma types while conserving as much normal tissue as possible. Tissue reconstruction using skin flaps and grafts is usually performed on the same day. However, for extensive skin cancers, care is coordinated through a multidisciplinary team of Mohs surgeons, head and neck surgeons, plastic surgeons, oculoplastic surgeons, and radiation oncologists. The eMohs system replaces a paper-based referral and follow-up system that traditionally supported communication among these groups of specialists. It includes a clinical solution that consists of state-of-the-art digital imaging devices, custom-made automated image- and document-processing modules, a dedicated Web server, HIPAA compliant communication protocols, and online self-training materials.

Other projects in the developmental stages include expansion of home-based monitoring for a variety of chronically ill patients by the Michigan Visiting Nurses Association and the substantial expansion of the chronic wound management program. Additionally, the TRC is providing technical support to the Department of Psychiatry for the development of a randomized pilot project pertaining to obsessive compulsive disorder, which will conduct patient consultations for 3 years. 
Technical support is also being provided to the Pediatric Physical Medicine and Rehabilitation Department for development of a Webbased intervention to set up a home program for arm exercises. The plan consists of building a Web-based data collection system and Web cams to monitor exercises. Finally, the TRC is also involved in assisting the Trauma Burn Center to create a secure Web site to share patient follow-up data with remote centers. This project includes enhancements to the internal communication regarding trauma case transfers and admissions as well as the secure transmission of relevant patient data to remote site physicians.

Currently and on an "as needed" basis, the TRC continues to provide a broad spectrum of necessary and required support services to programs designed to develop, implement, improve, monitor, and evaluate clinical telemedicine operations and professional remote consulting services. These include clinical telemedicine projects as well as related research and design projects and distance learning activities.

In summary, the primary functions of the Telemedicine Resource Center include the design of telemedicine programs (including equipment configuration, software and netware development, development of clinical protocols, analysis of patient flow, and Web design); providing technical assistance to clinicians and staff interested in exploring the use of these systems; serving as a point of contact for external parties interested in collaborating with the UMHS and negotiating business and financial terms involving appropriate departments, divisions, and offices within the system (for example, financial or legal offices and especially the clinical departments); providing on-demand and special training for interested faculty and staff; and providing technical assistance in the preparation of proposals for extramural funding, technical operations, maintenance, and ongoing support.

Historically, the TRC provided full videoconferencing support for all faculty and staff, both on demand and on a regularly scheduled basis, not only for telemedicine but also for all other educational, business, and managerial purposes. The aim was to substitute videoconferencing for travel across and beyond the med- ical campus for professional and business meetings. As the diffusion of proficiency in the use of videoconferencing technology increases among the staff, the TRC role will diminish in this regard, and become limited to training, equipment maintenance, and trouble shooting.

\section{OTHER MAJOR TELEMEDICINE RELATED INITIATIVES AT THE UMHS}

Several recent initiatives at the UMHS attest to both the vitality of this institution and to a serious commitment to use the capabilities of information technology (IT) to improve the efficiency and effectiveness of various aspects of patient care, education, and service. This bodes well for the institution because these technologies have substantial promise to enhance quality through greater conformity to standards of care and reduction of error, improving patient access, and possibly containing certain costs. At the same time, the proliferation of related and potentially overlapping initiatives and activities calls for a broader institutional assessment of commonalities as well as unique attributes among them and the creation of a coordinated and integrated structure to maximize the potential for efficiency and synergy. This is consistent with the leading strategic principles enunciated in the UMHS Strategic Direction 2005-2010.

\section{The Patient-Provider Portal Project}

This project was initiated in 2003 for the purpose of establishing a patient-provider portal to facilitate interactive communications among patients, providers, and staff. The patient portal enables and facilitates initial appointment requests, prescription renewal, self-help Webbased information, and integration with $\mathrm{M}$ Care. Plans include its expansion to referral requests, personal health records, lab/test results, disease management, e-visits, and other services. Initially, the physician portal will provide for referrals and access to clinical data. Subsequently, it will include secure messaging and education and information services. An additional M-CARE health insurance portal will provide information on benefits and claims, as well as personal health information tools. Em- 
ployer and provider portals will also be included in the latter.

\section{The Orders Management Project}

The major purpose of the Orders Management Project (OMP) initiated in 2004 is to improve patient safety and efficiency of care within the UMHHC. Its major elements include computerized prescriber order entry (CPOE), electronic medication administration documentation, nursing work list, and redundancy pharmacy system to reduce errors.

\section{LESSONS LEARNED}

A review of current and planned initiatives reveals several commonalities among them. In essence, they represent various and related applications of information technology in health care, including services for patients and services for providers, clinical decision support systems, and educational programming. They share not only a common denominator in the use of IT, but they also have common characteristics in some of the logistics for their implementation, in some constituent parts, and even in some specific activities. However, these initiatives must be coordinated, lest they result in several non-interoperable systems within the UMHS, reducing the potential overall efficiency and synergy in terms of their design, implementation, operation, and maintenance. Indeed, a coordinated and explicit policy pertaining to telemedicine and related projects would result in consistent procurement, system design, support, and maintenance, as well as common protocols for operations and accountability. Some institutions fail to utilize the available expertise within the institution, the creation of a common data base for research, and evaluation and adherence to a common strategy to serve institutional goals.

A substantial body of literature points to the fact that AHCs are facing increasing regulatory and market pressures, leading to revenue stream constraints relative to expenditures. To adhere to and not compromise their traditional missions of patient care, physician education/training, and research, AHCs must con- sider alternate strategies to improve the efficiency in the delivery of health services without compromising quality. In turn, AHCs have generally embraced the routine use of various forms of information technology in their operations, including telemedicine. The appeal of telemedicine in many instances derives from its potential to assist in addressing the basic challenges facing AHCs.

Examples cited here include providing specialist care to rural locations, best-practice protocol management in the home-care setting, and home-based monitoring for chronically ill patients. All of these initiatives address the mission of AHCs to provide specialist care to patients who need it, regardless of location. The challenge of access and the need to be cost-efficient were addressed in a telepsychiatry system for the commitment of mental patients and a dermatology triage system. Each opportunity to invest in a telemedicine initiative at the UMHS is assessed on its contribution to the mission, its ability to mitigate a business or a management challenge, or its potential longterm contribution to research and development.

Nationally, the approaches followed in the development and implementation of telemedicine programs vary from institution to institution, as have their relative success. The majority of AHCs (as well as other institutions and facilities) have relied on funding from extramural sources, such as the federal or state governments or alliances with other public institutions and private foundations. The advantages in these types of arrangements lie in the infusion of substantial funding and a relatively short timeline for project development and implementation. The major disadvantages associated with many such funding arrangements include an inappropriate quick start and the lack of a demonstrable clinical and financial need and, therefore, the lack of mainstream support. Indeed, short-term funding for such projects may preclude long term sustainability, maturation, and systematic evaluation. It may also preclude institutional adoption of telemedicine in its operational infrastructure.

As detailed here, the UMHS telemedicine program evolved almost wholly on the basis of 
clinical faculty interest in extending their reach to serve patients requiring their expertise, but having limited accessibility due to relative location or other access disadvantages. It has also been implemented to control, to the extent possible, the mix and volume of in- and outpatients admitted to the UMHHC. Furthermore, it was developed in a gradual and deliberative "grass roots" fashion, guided exclusively by the mutual interest and realized needs of the clinical faculty and administration. Telemedicine solutions were pursued only after problems were identified and the suitability of the telemedicine solutions was assessed and determined to be reasonable.

Of particular interest has been the independence of the UMHS telemedicine development from extramural funding. The great majority of funding has been through funds provided by the Faculty Group Practice (for targeted projects) and the UMHHC (for ongoing operations) and funneled through the TRC. This intramural funding has assured appropriate long-term support for telemedicine programs, thus ensuring time for program maturation, evaluation, and, when demonstrated, sustained support. The intramural funding also provides for flexibility in project/program development not usually associated with extramurally funded projects and contracts.

Finally, there is a clear allocation of responsibility and division of labor between the TRC and the UMHS clinical departments. The TRC provides a wide spectrum of services as needed in the development, funding, and implementation of telemedicine programs and projects. At the same time, the plan is to decentralize telemedicine activities and to place them in their respective clinical and educational services, while the TRC remains ready to assist when needed.

\section{REFERENCES}

1. Telemedicine Information Exchange. (Available at http://tie.telemed.org/programs_t2/browsebyaffiliation_t2.asp, 2005.)
2. Freburger J, Hurley R. Academic health centers and the changing health care market. Med Care Res Rev 1999;56:277-306.

3. Garson A, Levin S. Ten 10-year trends for the future of healthcare: implications for academic health centers. The Ochsner J 2001;3:10-15.

4. Retchin S, Clark R. Contemporary challenges and opportunities at academic health centers. I Healthcare Manag 2005;50:121-135.

5. Institute of Medicine. Definition of serious and complex medical conditions. Chrvala C, Sharfstein S, eds. Washington, DC: National Academy Press, 2000. (Also available at http://www.nap.edu/books/ 0309066409/html/ Last accessed 9/23/05.)

6. Medical Post. Volume 36, Issue 22, June 13, 2000.

7. Eisenberg L. Marketplace medicine: $\mathrm{Rx}$ for disaster. Academe 1999;85:26-30. (Also available at http:// www.aaup.org/publications / Academe/1999/99nd/ ND99Eise.htm/ Last accessed 9/23/05.)

8. Reinhardt U. Grow your own for-profit philanthropist. (Available at http://64.233.167.104/search? $\mathrm{q}=$ cache:hrBwAQSqaKgJ:conferences.mc.duke.edu/ privatesector/dpsc1995/grow.htm + Patient + mix + Academic + Medical + Center\&hl $=\mathrm{en} /$, 1995. Last accessed 9/23/05.)

9. Badgett J. General pediatric teaching clinics and managed care. Pediatrics 1998;101:775-778.

10. Weitekamp M, Thorndyke L, Evearts C. Planning for academic health centers. Am J Med 1996;3:309-315.

11. UMHS. UMHS Mission Strategic Direction 2005-2010. (Available at http://www.med.umich.edu/strategic/, 2005.)

12. UMHHC FY06 Operating Budget. Version 8.2, May 3, 2005, and Strategic Financial and Capital Plan, January, 2005.

13. Bagust A, Place M, Posnett J. Dynamics of bed use in accommodating emergency admissions: stochastic simulation model. Br Med I 1999;319:155-158.

14. Bazzoli G, Brewster L, Liu G, et al. Does U.S. hospital occupancy need to be expanded? Health Affairs 2003;22:40-54.

15. Alessi, N. Quantitative documentation of the therapeutic efficacy of adolescent telepsychiatry, Telemed J e-Health 2003;9:283-290.

Address reprint requests to: Gary Shannon, Ph.D. Department of Geography 1449 Patterson Office Tower University of Kentucky Lexington, KY 40506-0027

E-mail: gwshan00@uky.edu 
This article has been cited by:

1. Sanjeev Arora, Cynthia M. A. Geppert, Summers Kalishman, Denise Dion, Frank Pullara, Barbara Bjeletich, Gary Simpson, Dale C. Alverson, Lori B. Moore, Dave Kuhl, Joseph V. Scaletti. 2007. Academic Health Center Management of Chronic Diseases through Knowledge Networks: Project ECHO. Academic Medicine 82:2, 154-160. [CrossRef] 